\title{
Improved fatty acid profiles in seeds of Camelina sativa by artificial microRNA mediated FATB gene suppression
}

\section{Authors: Mehmet Ozseyhan, Pengcheng Li, GunNam $\mathrm{Na}$, Zhenjing Li, Changlu Wang, and Chaofu Lu}

NOTICE: this is the author's version of a work that was accepted for publication in Biochemical \& Biophysical Research Communications. Changes resulting from the publishing process, such as peer review, editing, corrections, structural formatting, and other quality control mechanisms may not be reflected in this document. Changes may have been made to this work since it was submitted for publication. A definitive version was subsequently published in Biochemical \& Biophysical Research Communications, VOL\# 503, ISSUE\# 2, (September 2018) DOI\# 10.1016/ j.bbrc.2018.06.051.

Ozseyhan, Mehmet, Pengcheng Li, GunNam Na, Zhenjing Li, Changlu Wang, and Chaofu Lu. "Improved fatty acid profiles in seeds of Camelina sativa by artificial microRNA mediated FATB gene suppression." Biochemical \& Biophysical Research Communications 503, no. 2 (September 2018): 621-624. DOI:10.1016/j.bbrc.2018.06.051.

Made available through Montana State University's ScholarWorks

scholarworks.montana.edu 


\title{
Improved fatty acid profiles in seeds of Camelina sativa by artificial microRNA mediated FATB gene suppression
}

\author{
Mehmet E. Ozseyhan ${ }^{\mathrm{a}}$, Pengcheng Li ${ }^{\mathrm{b}}$, GunNam Na ${ }^{\mathrm{a}}$, Zhenjing Li ${ }^{\mathrm{b}}$, Changlu Wang ${ }^{\mathrm{b}}$, \\ Chaofu Lu ${ }^{\text {a, * }}$ \\ a Department of Plant Sciences and Plant Pathology, Montana State University, Bozeman, MT 59717, USA \\ ${ }^{\mathrm{b}}$ College of Food Engineering and Biotechnology, Tianjin University of Science and Technology, Tianjin 300222, China
}

\begin{abstract}
A B S T R A C T
The fatty acid profile of plant oils determines their quality and uses. Saturated fatty acids are often not desirable from the standpoints of nutrition and some industrial applications. Camelina sativa is a reemerged oilseed crop, however its oil needs to be improved to meet different application requirements. In this study, saturated fatty acids were greatly reduced by down-regulating genes encoding the fatty acyl-ACP thioesterases (FATB). An artificial microRNA (amiFATB) was created by replacing a microRNA sequence in the camelina Csa-miR159a gene with a FATB gene specific sequence. Seed-specific expression of amiFATB caused a $45 \%$ reduction of palmitic acid (16:0) and a $38 \%$ reduction of stearic acid (18:0) compared to wildtype seeds. The total saturated fatty acid content was decreased by $35 \%$ from $14.6 \%$ to $9.4 \%$ of total fatty acids. When amiFATB was expressed in a high-oleic acid transgenic line, it caused further increased oleic acid content. This work demonstrates that the FATB genes in camelina can be effectively knocked down by an artificial microRNA targeting gene-specific sequences, thus provides an additional tool to improve seed oils for desired properties.
\end{abstract}

\section{Introduction}

Vegetable oils are an important source of food and feed, and increasingly industrial feedstocks such as biofuels [1]. To provide desired functionalities and nutritional attributes, genetic engineering is being conducted to improve fatty acid composition by manipulating key genes involved in lipid metabolism. In oilseeds, newly synthesized fatty acids are exported from plastids mainly in the form of the monounsaturated oleic acid (18:1) along with smaller amounts of the saturated 16:0 and 18:0. These fatty acids are released from acyl-acyl carrier protein (acyl-ACP) by two different classes of thioesterases (FAT). The FATA has a higher activity on 18:1 while the FATB prefers saturated substrates [2]. A large proportion of oleic acid is modified before being incorporated into triacylglycerol through either desaturation by the desaturases FAD2 and FAD3 acting on the phosphatidylcholine (PC) substrates to form the polyunsaturated linoleic (18:2) and linolenic (18:3) acids, or elongation to form $20: 1$ and $22: 1$ by the fatty acid elongase FAE1 [3]. Controlling these metabolic steps may effectively improve plant oils for required nutritional or industrial properties $[4,5]$.

Camelina (Camelina sativa) is a promising oilseed crop that is under intensive development mainly for bioenergy production. An important limitation for camelina is the suboptimal quality of its oil [6], which contains high contents of undesirable polyunsaturated (50\%-60\% of the total fatty acid), saturated ( $10 \%)$, and the verylong chain $(\sim 12 \%)$ fatty acids [7], which are known to be associated with low oxidative stability, poor cold flow and high melting point, respectively, of the biodiesel [8]. Previously, we have successfully decreased polyunsaturated and very-long-chain fatty acids by suppression or deletion of FAD2 and FAE1 genes in camelina using RNA interference (RNAi) or CRISPR/Cas9 technologies $[7,9,10]$. The objective of this study is to decrease saturated fatty acids and to increase oleic acid. Mutations or knockdown of FATB genes have been shown to be associated with low saturates in seed oils such as canola and soybean [11,12]. Here, we used a microRNA mediated approach to downregulate the FATB genes in camelina. MicroRNAs are short 20-24 nucleotide non-coding RNA sequences. They control gene expression by binding to specific complementary mRNA targets in the untranslated regions or the coding sequence, 
leading to mRNA degradation or translational inhibition [13]. Such post-transcriptional gene silencing mechanisms may be used in plants by antisense expression or hairpin-based RNA interference (RNAi), which produce miRNAs to target transcripts of any genes of interest. It is also possible to modify miRNA sequences and create artificial miRNAs to target the gene transcripts alter gene expression and modify seed oil composition [14]. Here we demonstrated that FATB genes in camelina were effectively downregulated by an artificial microRNA159a that led to changed fatty acid composition in seed.

\section{Material and methods}

\subsection{Plant materials}

Camelina ( $C$ sativa) cv. Suneson, and a previously created higholeic transgenic plant (HiOle1) [9] were used to transform the FATB artificial microRNA gene. Plants were grown in a greenhouse at $22^{\circ} \mathrm{C}$ under natural light conditions supplemented with highpressure sodium lights with a $16 \mathrm{~h}$ photoperiod ( $16 \mathrm{~h}$ of light and $8 \mathrm{~h}$ of darkness). Young leaves, stems, inflorescence, and developing seeds at 16 days after flowering were harvested, chilled in liquid nitrogen, then stored at $-80^{\circ} \mathrm{C}$. Seedlings and roots were harvested from plants grown under sterile conditions on MS medium (PhytoTechnology Laboratories, Shawnee Mission, KS).

\section{Vector construction and plant transformation}

The mature sequence in the camelina microRNA (Csa-miR159a) was replaced by a 21 mer FATB artificial microRNA sequence identified by the WMD3 software (http://wmd3,weigelworld,org/cgibin/webapp.cgi) [15]. Initially, Arabidopsis FATB gene sequence was used to identify the amiFATB candidates that were targeted by Ath-miR159a without affecting off-target genes. The amiFATB sequences were then compared with the camelina FATB sequences to choose one that matched all three homologous copies. The fragment of the Csa-miR159a stem loop with amiFATB sequence was synthesized by GeneWiz (South Plainfield, NJ), and was inserted into a binary vector, pPhas-Gly-DsRed2 [9], between the EcoRI and Xhol sites so that amiFATB was put under the seed-specific glycinin promoter. This construct contains a DsRed selection marker for easy isolation of transgenic seeds by fluorescence [16]. Sequences of miRNA and amiRNAs are listed in Table S1.

\subsection{Gene expression analysis by $R T-P C R$}

Total RNA was isolated using Norgen miRNA extraction BioKit (Thorold, ON, Canada), measured by NanoDrop (Nanodrop Technologies, Wilmington, DE, USA). For the qRT-PCR or semi-qRT-PCR, the synthesis of first-strand cDNA was performed using SuperScript III reverse transcriptase (Wal tham, MA, USA). The primer pairs used for the PCR are listed in Table S1. For the qRT-PCR of Csa-miR159a, cDNAs were synthesized using methods described previously [17]. Amplification and quantification of CDNA was done with the CFX96 Real-time system from Bio-Rad and PerfeCTa SYBR Green FastMix from Quantabio (Beverly, MA, USA). The following standard thermal profile was used for PCR: $95^{\circ} \mathrm{C}$ for $5 \mathrm{~min}$, 40 cycles of $95^{\circ} \mathrm{C}$ for $5 \mathrm{~s}, 60^{\circ} \mathrm{C}$ for $10 \mathrm{~s}, 72^{\circ} \mathrm{C}$ for $1 \mathrm{~s}$ and melting curve analysis.

\subsection{Fatty acid analysis}

Camelina seeds were crushed and lipids were transmethylated according to procedures described previously [18]. The resulting fatty acid methyl esters (FAMEs) were analyzed in a gas chromatograph (GC) (Shimadzu Scientific Instruments, Columbia, MD) fitted with a $15 \mathrm{~m} \times 0.25 \mathrm{~mm}$ column (DB-23; Agilent, Lexington, MA) and a flame ionization detector (FID). The GC was programmed for an initial temperature of $180^{\circ} \mathrm{C}$ for $1 \mathrm{~min}$ followed by an increase of $10^{\circ} \mathrm{C} / \mathrm{min}$ to $230^{\circ} \mathrm{C}$ and maintained for a further $1 \mathrm{~min}$.

\section{Results}

\subsection{Generation of transgenic plants expressing an artificial microRNA}

Camelina has three highly similar FATB genes (Csa17g011970, Csa14g009990, Csa03g011990) [19]. To ensure effective knockdown of these genes, a 21mer artificial microRNA sequence was designed that targeted all three FATB coding sequences (Table S1). The Arabidopsis Ath-miR159 has been used previously to successfully induce gene silencing in Arabidopsis [14]. Previously, we detected an Ath-miR159 homolog named Csa-miR159 in camelina [20]. To determine whether Csa-miR159 is actively expressed in camelina, we carried out an RT-PCR experiment using RNA extracted from different tissues including young leaf, stem, root and developing seed (16 days after flowering). As shown in Fig. 1, Csa-miR159 transcripts were detected in all tissues examined. The highest expression level was detected in developing seeds. We reasoned that Csa-miR159 was able to express the FATB artificial microRNA (amiFATB) in camelina. The amiFATB sequence was inserted into a camelina microRNA gene (Csa-miR159a) to replace the native mature miRNA sequence. A construct was made by inserting the modified stem-loop region of the Csa-miR159a gene under a seedspecific glycinin promoter in the pPhas-Gly-DsRed vector (Fig. S1). The amiFATB construct was transformed into an agrobacterium strain (GV3101) and then into the camelina wildtype Suneson plants. We obtained 16 transgenics seeds selected by using the DsRed fluorescent marker [16].

\section{Reduction of saturated fatty acids in the FATB-knockdown seeds}

All transgenic amiFATB plants were grown to the $\mathrm{T} 3$ generation, and the homozygosity was determined by all-red seeds conferred by the DsRed marker. To examine the expression levels of FATB genes, developing seeds at 16 days after flowering were harvested from Suneson and 4 independent homozygous amiFATB lines. RNA was extracted, and RT-PCR was performed using primers that

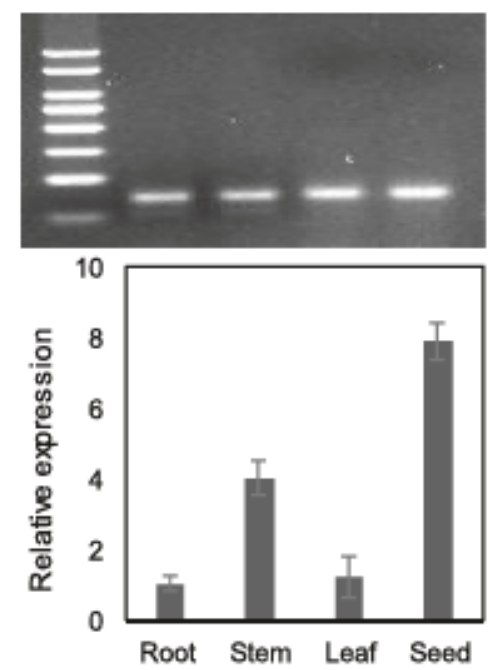

Hg. 1. Quantitative RT-PCR results of the Csa-miR159a gene in camelina tissues. 
A

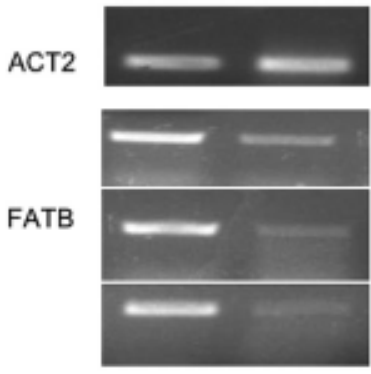

Suneson amiFATB
B

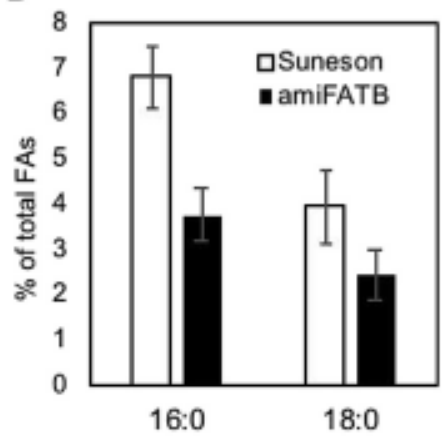

Hg. 2. The effect of amiFATB on FATB expression and saturated fatty acids accumulation. (A) RT-PCR detection of FATB in amiFATB transgenics and Suneson. The actin (ACT2) was used as a control gene. (B) The levels of 16:0 and 18:0 in Suneson and amiFATB seeds.

amplified all three homologous FATB genes (Table S1). The results showed that FATB transcript levels were significantly lower in transgenic lines than in the wildtype (Fig. 2A). This indicated that the amiFATB transgene has effectively knocked down FATB genes in camelina developing seed.

To assess the effect of the amiFATB transgene on fatty acid metabolism in camelina, fatty acid composition was determined in mature T3 seeds by gas chromatography. The results showed that levels of saturated fatty acids were decreased in the amiFATB seeds compared to those in the non-transgenic Suneson. Especially, a $45 \%$ reduction of $16: 0$ and a $38 \%$ reduction of $18: 0$ were observed in amiFATB compared to Suneson (Fig. 2B). This result was consistent with the enzyme substrate specificity of FATB on saturated $(16: 0$ and 18:0) acyl-ACPs in plastids [2]. Other saturated fatty acids (20:0 and 22:0) were also slightly decreased, consequently the total saturated fatty acids were decreased from $14.6 \%$ in Suneson to $9.4 \%$ in amiFATB seeds, about $35 \%$ reduction compared with nontransgenic seeds (Table 1 ).

\subsection{The effect of FATB-knockdown in a high-oleic line}

Corresponding to decreased saturated fatty acids, the levels of oleic acid and other unsaturated fatty acids were slightly increased in the amiFATB seeds (Table 1). Previously, we created transgenic camelina lines that accumulated high levels of oleic acid by antisense or hairpin mediated RNAi knockdown on FAD2, FAD3 and FAE1 genes [7,9]. To explore whether down-regulating FATB expression may further increase the oleic acid content, we transformed the amiFATB construct into a high-oleic line (HiOle1) containing the FAD2-FAD3-FAE1 RNAi construct and a herbicide resistance marker [9]. Twenty transgenic lines were obtained that contained both herbicide and DsRed selection markers. Consequently, six T3 HOamiFATB lines that showed clearly decreased FATB transcripts determined by semi-qRT-PCR were analyzed for their seed fatty acid composition. The results showed that saturated fatty acids (16:0, 18:0 and 20:0) were decreased as expected. The

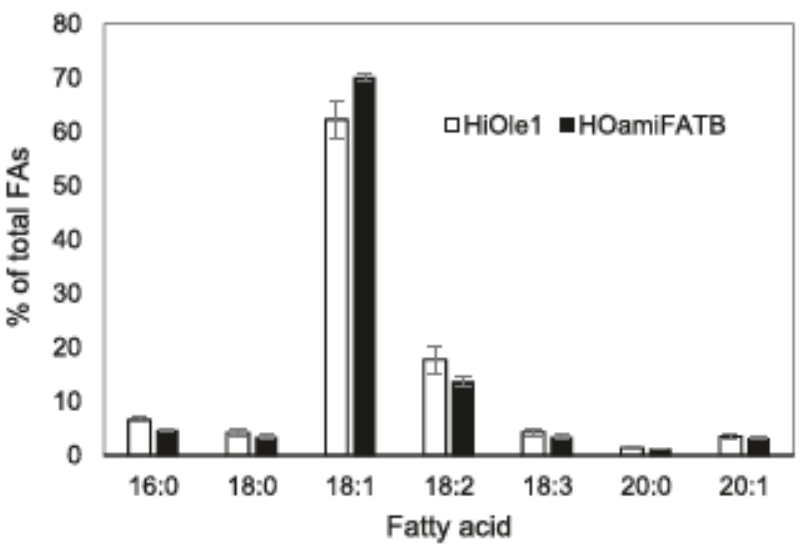

Fig. 3. A comparison of seed fatty acid profiles between a high-oleic line with its FATB knockdowns.

composition of unsaturated fatty acids was also changed. Significantly, the oleic acid was increased from about $62 \%$ to over $70 \%$ (Fig. 3).

\section{Discussion}

The fatty acyl-ACP thioester FATB is a major determinant for saturated fatty acid accumulation in oilseeds. Low palmitate (16:0) in soybean was associated with the mutation of a FATB gene [11], and the Arabidopsis fatb knockout mutant accumulated only $50 \%$ saturated fatty acids of the wild type level [21]. In this study, we demonstrated that seed-specific expression of an artificial microRNA (amiFATB) significantly decreased FATB gene transcripts in developing seeds and caused about $45 \%$ and $38 \%$ reduction of $16: 0$ and 18:0, respectively, in camelina seed oil. The reduction of the saturated fatty acid content in amiFATB was at a similar level found in the Arabidopsis fatb mutant [21]. In tissues of the fatb plant, the remaining about $50 \%$ saturated fatty acids were presumably synthesized by other genes including the plastidial glycerol-3phosphate:acyl-ACP acyltransferase (ACT1). Significantly, plant growth of the fatb and fatb/act1 mutants were severely retarded, indicating that saturated fatty acids are essential for normal plant growth [21]. It is therefore infeasible to generate the FATB knockout camelina plants, which may affect plant growth while not be able to further reduce saturated fatty acids in seed. We did not observe abnormal seed germination and plant growth of the amiFATB lines.

A major goal of oilseed breeding is to increase the desired oleic acid content. Our results indicated that FATB knockdown not only decreased saturated fatty acids, but also caused changes in unsaturated fatty acids especially enhanced oleic acid accumulation. Simultaneous downregulation of FATB and FAD2 in soybean has been shown to increase oleic acid and decrease palmitic acid [22]. Similarly, expression of amiFATB in a FAD2-FAD3-FAE1 RNAi line also increased oleic acid in camelina. Besides decreased saturates, the increased oleic acid in the HOamiFATB seeds seemed to be also at the expenses of linoleic acid (18:2). It is not clear whether reduced

Table 1

Fatty acid composition in Suneson and amiFATB seeds.

\begin{tabular}{|c|c|c|c|c|c|c|c|c|c|c|c|c|}
\hline & & $16: 0$ & $18: 0$ & $18: 1$ & $18: 2$ & $18: 3$ & $20: 0$ & $20: 1$ & $20: 2$ & $20: 3$ & $22: 0$ & $22: 1$ \\
\hline \multirow[t]{2}{*}{ Suneson } & Ave & 6.8 & 3.9 & 11.5 & 18.8 & 36.4 & 3.3 & 13.0 & 1.7 & 1.3 & 0.5 & 2.8 \\
\hline & SD & 0.7 & 0.8 & 0.7 & 2.1 & 2.6 & 0.7 & 1.1 & 0.2 & 0.2 & 0.1 & 0.4 \\
\hline \multirow[t]{2}{*}{$\operatorname{amiFATB}^{2}$} & Ave & 3.8 & 2,4 & 13.4 & 18.9 & 37.5 & 2.8 & 13.9 & 1.8 & 1.3 & 0.4 & 3.5 \\
\hline & SD & 0.6 & 0.5 & 1.6 & 1.6 & 4.0 & 0.4 & 0.6 & 0.1 & 0.2 & 0.1 & 0.5 \\
\hline
\end{tabular}

Data represent average and standard deviation from 4 independent lines. 
flux of 16:0 and 18:0 into the cytosolic triacylglycerol biosynthesis pathway or the FATB gene knockdown affected FAD2 activity in the RNAi line. Nevertheless, our results and previous studies [22] suggest that simultaneous knockdown of genes encoding key fatty acid modification enzymes including FATB, FAD2, FAD3 and FAE1 may enhance accumulation of oleic acid in oilseeds. Further increasing oleic acid in HOamiFATB seeds may be achieved by eliminating the very-long-chain fatty acids (20:0 and 20:1) by deleting FAE1 genes. It was possible to knockout FAE1 genes in camelina by the CRISPRCas9 technology, which blocked fatty acyl flux into the elongation pathway and resulted in enhanced accumulation of 18:1 and its derived polyunsaturated fatty acids (18:2 and 18:3) [10]. Unlike FAE1 which can be deleted without causing abnormal plant growth, FATB and FAD2 are essential for normal plant growth [21,23,24]. Our results provide an additional tool to effectively downregulate gene expression by artificial microRNAs. Combining this with other biotechnological tools for gene silencing will allow for the development of high-oleic camelina for improved oil qualities.

\section{Conflicts of interest}

The authors declare no conflict of interest.

\section{Acknowledgements}

This research was funded by the Office of Science (BER), US Department of Energy, grant no. DE-SC0016400, the US National Science Foundation, grant no. DBI-1339358, and the USDA National Institute of Food and Agriculture Hatch project no. 1015638.

\section{Appendix A. Supplementary data}

Supplementary data related to this article can be found at https://doi.org/10.1016/j.bbrc.2018.06.051.

\section{References}

[1] C. Lu, J.A. Napier, T.E. Clemente, E.B. Cahoon, New frontiers in oilseed biotechnology: meeting the global demand for vegetable oils for food, feed, biofuel, and industrial applications, Curr. Opin. Biotechnol. 22 (2011) $252-259$.

[2] J.J. Salas, J.B. Ohlrogge, Characterization of substrate specificity of plant FatA and FatB acyl-ACP thioesterases, Arch. Biochem. Biophys. 403 (2002) 25-34.

[3] J. Ohlrogge, J. Browse, Lipid biosynthesis, Plant Cell 7 (1995) 957-970.

[4] A.J. Kinney, Designer oils for better nutrition, Nat. Biotechnol. 14 (1996) 946

[5] T.P. Durrett, C. Benning, J. Ohlrogge, Plant triacylglycerols as feedstocks for the production of biofuels, Plant J. 54 (2008) 593-607.

[6] C. Ciubota-Rosie, J.R. Ruiz, M.J. Ramos, Á. Pérez, Biodiesel from Camelina sativa: a comprehensive characterisation, Fuel 105 (2013) 572-577.

[7] J. Kang, A.R. Snapp, C. Lu, Identification of three genes encoding microsomal oleate desaturases (FAD2) from the oilseed crop Camelina sativa, Plant Physiol. Biochem. 49 (2011) 223-229.

[8] G. Knothe, "Designer" biodiesel: optimizing fatty ester composition to improve fuel properties, Energy Fuel. 22 (2008) 1358-1364.

[9] H.T. Nguyen, J.E. Silva, R. Podicheti, J. Macrander, W. Yang, T.J. Nazarenus, J.W. Nam, J.G. Jaworski, C. Lu, B.E. Scheffler, K. Mockaitis, E.B. Cahoon, Camelina seed transcriptome: a tool for meal and oil improvement and translational research, Plant Biotechnol. J 11 (2013) 759-769.

[10] M.E. Ozseyhan, J. Kang, X. Mu, C. Lu, Mutagenesis of the FAE1 genes significantly changes fatty acid composition in seeds of Camelina sativa, Plant Physiol. Biochem. (Paris) 123 (2018) 1-7.

[11] A.J. Cardinal, J.W. Burton, A.M. Camacho-Roger, J.H. Yang, R.F. Wilson, R.E. Dewey, Molecular analysis of soybean lines with low palmitic acid content in the seed oil, Crop Sci. 47 (2007) 304-310.

[12] A. Pandian, Q. Liu, C. Hurlestone, S. Singh, P. Salisbury, A. Green, Development of nutritionally superior Brassica napus and B. juncea oils using RNAi-mediated gene silencing, in: 4th International Crop Science Congress. Brisbane Australia, Sept, 2004.

[13] D.P. Bartel, MicroRNAs: target recognition and regulatory functions, Cell 136 (2009) 215-233.

[14] O. Stephan, S. Rebecca, W. Detlef, Gene silencing in plants using artificia microRNAs and other small RNAs, Plant J. 53 (2008) 674-690.

[15] R. Schwab, S. Ossowski, M. Riester, N. Warthmann, D. Weigel, Highly specific gene silencing by artificial MicroRNAs in Arabidopsis, Plant Cell 18 (2006) $1121-1133$.

[16] C. Lu, J. Kang, Generation of transgenic plants of a potential oilseed crop Camelina sativa by Agrobacterium-mediated transformation, Plant Cell Rep. 27 (2008) 273-278.

[17] E. Varkonyi-Gasic, R. Wu, M. Wood, E.F. Walton, R.P. Hellens, Protocol: a highly sensitive RT-PCR method for detection and quantification of microRNAs, Plant Meth. 3 (2007) 12.

[18] C. Lu, M. Fulda, J.G. Wallis, J. Browse, A high-throughput screen for genes from castor that boost hydroxy fatty acid accumulation in seed oils of transgenic Arabidopsis, Plant J. 45 (2006) 847-856.

[19] M.F. Rodríguez-Rodríguez, J.J. Salas, R. Garcés, E. Martínez-Force, Acyl-ACP thioesterases from Camelina sativa: cloning, enzymatic characterization and implication in seed oil fatty acid composition, Phytochemistry 107 (2014) $7-15$

[20] S. Poudel, N. Aryal, C. Lu, Identification of microRNAs and transcript targets in Camelina sativa by deep sequencing and computational methods, PLoS One 10 (2015) e0121542.

[21] G. Bonaventure, J.J. Salas, M.R. Pollard, J.B. Ohlrogge, Disruption of the FATB gene in Arabidopsis demonstrates an essential role of saturated fatty acids in plant growth, Plant Cell 15 (2003) 1020-1033.

[22] B. Tony, S. Shirley, E. Farida, X. Aiqiu, Z. You, M. Michelle, S. Bruce, K. Anthony S. Paul, Ribozyme termination of RNA transcripts down-regulate seed fatty acid genes in transgenic soybean, Plant J. 30 (2002) 155-163.

[23] M. Miquel, D. James Jr., H. Dooner, J. Browse, Arabidopsis requires polyunsaturated lipids for low-temperature survival, Proc. Natl. Acad. Sci. U.S.A. 90 (1993) 6208-6212.

[24] C. Morineau, Y. Bellec, F. Tellier, L. Gissot, Z. Kelemen, F. Nogue, J.D. Faure, Selective gene dosage by CRISPR-Cas9 genome editing in hexaploid Camelina sativa, Plant Biotechnol. J 15 (2017) 729-739. 